\title{
Study of the conformal hyperscaling relation through the Schwinger-Dyson equation
}

\section{Yasumichi Aoki ${ }^{a}$, Tatsumi Aoyama ${ }^{a}$, Masafumi Kurachi ${ }^{* a}$, Toshihide Maskawa ${ }^{a}$, Kei-ichi Nagai ${ }^{a}$, Hiroshi Ohki ${ }^{a}$, Akihiro Shibata ${ }^{b}$, Koichi Yamawaki $^{a}$ and Takeshi Yamazaki $^{a}$}

\section{LatKMI Collaboration}

${ }^{a}$ Kobayashi-Maskawa Institute for the Origin of Particles and the Universe (KMI), Nagoya University, Nagoya 464-8602, Japan

${ }^{b}$ Computing Research Center, High Energy Accelerator Research Organization (KEK), Tsukuba 305-0801, Japan

E-mail: kurachi@kmi.nagoya-u.ac.jp

\begin{abstract}
We formulate the Schwinger-Dyson (SD) equation in a finite-size space-time for the study of the correction to the finite-size hyperscaling relation. By using data obtained from the finitevolume SD equation, we show that the mass anomalous dimension, when identified through the finite-size hyperscaling relation neglecting the mass corrections as is often done in the lattice analyses, yields a substantially lower value than its value at the infrared fixed point. From the analytical expression of the solution of the ladder SD equation, we identify the form of the leading correction to the hyperscaling relation. We also applied the finite-volume SD equation to the chiral-symmetry-breaking phase and found that when the theory is close to the critical point such that the dynamically generated mass is much smaller than the explicit breaking mass, the finitesize hyperscaling relation is still operative.
\end{abstract}

The 30th International Symposium on Lattice Field Theory

June 24 - 29, 2012

Cairns, Australia

${ }^{*}$ Speaker. 


\section{Introduction}

Technicolor (TC) model [1, 2] is an attractive candidate for the dynamical origin of the Electroweak symmetry breaking. Especially, a model based on dynamics which has an approximate infrared fixed point (IRFP) with large mass anomalous dimension (so-called Walking Technicolor model [3, 4]) is considered to be phenomenologically viable. Large $N_{f}$ QCD, which is an SU(3) gauge theory with many number $\left(N_{f}\right)$ of massless fermions, is expected to possess such property. From the analysis based on the Schwinger-Dyson (SD) equation with the two-loop running coupling, the critical number of flavor, above which the chiral symmetry restores and the theory possesses an IRFP, is estimated as $N_{f}^{\mathrm{cr}} \simeq 11.9$. Since a viable Walking TC model resides just below the critical point, precise determination of $N_{f}^{\mathrm{cr}}$ by using fully non-perturbative method, like lattice gauge theory, is of great importance.

One of the methods to judge whether a theory is infrared conformal or not from lattice data is using the finite-size hyperscaling relation of a mass-deformed conformal gauge theory [5]:

$$
M=L^{-1} f(x) .
$$

Here, $M$ is a physical quantity such as meson mass or decay constant, $L$ is the size of space and time, and $f$ is some function of scaling variable $x . x$ is defined as $x \equiv \hat{L} \hat{m}_{0}^{1 /\left(1+\gamma_{m}^{*}\right)}$, where $m_{0}$ is the bare fermion mass and $\gamma_{m}^{*}$ is the mass anomalous dimension at the IRFP. Here, we introduced dimensionless quantities, $\hat{L} \equiv L \Lambda$ and $\hat{m}_{0} \equiv m_{0} / \Lambda$, where we take $\Lambda$ as the UV scale at which the infrared conformality terminates. (This $\Lambda$ is same as the one defined in Ref. [6]) In the case of lattice simulations, it is usually taken to be the inverse lattice spacing $a^{-1}$. Since the above scaling relation is satisfied only when the theory is infrared conformal, one can judge whether a candidate theory possesses an IRFP or not by measuring the low-energy quantities on the lattice for various combination of input values of $\hat{L}$ and $\hat{m}_{0}$, then checking whether Eq. 1.1) is satisfied for a certain value of $\gamma_{m}^{*}$.

There are a couple of questions to be raised here regarding use of this finite-size hyperscaling relation for the study of infrared conformality: How small $m_{0}$ has to be so that the hyperscaling relation is approximately satisfied? (Remember that the bare fermion mass, $m_{0}$, which is introduced as a probe, itself necessarily breaks the infrared conformality of the original theory.) What is the form of correction if it is not small enough? When the theory in question does not have an IRFP (namely, in the phase where the chiral symmetry is spontaneously broken) in the first place, how and how much is the hyperscaling relation violated?

$\mathrm{SD}$ equation is a useful tool for studying such questions. From the solution of the SD equation with a certain approximation, for a given theory (in the continuum, infinite-volume space-time), we know whether the chiral symmetry is broken or not, and the value of $\gamma_{m}^{*}$ as well. As we will explain later, the SD equation can be formulated in a finite-volume space-time, and numerical calculations can be easily done for a wide range of parameter space of $\left(\hat{L}, \hat{m}_{0}\right)$. By doing finite-size hyperscaling analysis with using "data" generated by solving the finite-volume SD equation, and comparing the result obtained from that analysis to the "answer" which is known from the solution of the infinitevolume SD equation, we can study the reliability of the finite-size hyperscaling analysis itself in a self consistent manner. To a certain extent, even analytical understanding can be obtained regarding the finite-mass correction to the hyperscaling relation, which we will mention later as well. 


\section{Hyperscaling analysis through the SD equation with a finite-size space-time}

$\mathrm{SD}$ equation is the self-consistent equation for the full fermion propagator, $i S_{F}^{-1} \equiv A(p) p-$ $B(p)$. To put the SD equation in a finite space-time, all one needs to do is to introduce the discrete momentum variables: $\tilde{p}_{i}=\frac{2 \pi n_{i}}{L}$, where $i$ indicates the $i$-th component of the momentum variable, and $n_{i}$ 's are integers which label discrete momentum variables [7].

$$
\begin{aligned}
& \tilde{A}(\tilde{p})=1+ \frac{1}{L^{4}} \sum_{\tilde{k}} \frac{C_{2} \bar{g}^{2}\left((\tilde{p}-\tilde{k})^{2}\right) \tilde{A}(\tilde{k})}{\tilde{k}^{2} \tilde{A}(\tilde{k})^{2}+\tilde{B}(\tilde{k})^{2}} \\
& \cdot\left[\frac{(\tilde{p} \cdot \tilde{k})}{\tilde{p}^{2}(\tilde{p}-\tilde{k})^{2}}+2 \frac{\{\tilde{p} \cdot(\tilde{p}-\tilde{k})\}\{\tilde{k} \cdot(\tilde{p}-\tilde{k})\}}{\tilde{p}^{2}(\tilde{p}-\tilde{k})^{4}}\right], \\
& \tilde{B}(\tilde{p})=m_{0}+\frac{1}{L^{4}} \sum_{\tilde{k}} \frac{3 C_{2} \bar{g}^{2}\left((\tilde{p}-\tilde{k})^{2}\right)}{\tilde{k}^{2} \tilde{A}(\tilde{k})^{2}+\tilde{B}(\tilde{k})^{2}} \frac{\tilde{B}(\tilde{k})}{(\tilde{p}-\tilde{k})^{2}} .
\end{aligned}
$$

Here, $C_{2}$ is the quadratic Casimir, and $\bar{g}\left((p-q)^{2}\right)$ is the running coupling constant. The improved ladder approximation is adopted in the above expression. We use the following form of the running coupling as an approximation of the two-loop running coupling of the large $N_{f}$ QCD:

$$
\alpha\left(\mu^{2}\right) \equiv \frac{\bar{g}^{2}\left(\mu^{2}\right)}{4 \pi}=\alpha_{*} \theta\left(\Lambda^{2}-\mu^{2}\right)
$$

Here, $\alpha^{*}$ is the value of the two-loop running coupling at the IRFP. The mass function, $\Sigma(\tilde{p}) \equiv$ $B(\tilde{p}) / A(\tilde{p})$, is obtained from the solutions of Eqs. (2.1) and (2.2), then so called the "pole mass," $m_{P}$, which is defined by $\Sigma\left(\sqrt{\tilde{p}^{2}}=m_{P}\right)=m_{P}$ can be calculated from it. Since it is known, from the study with the Bethe-Salpeter equation [8], that $m_{P}$ is proportional to meson masses, we use $m_{P}$ as low-energy physical quantity which appears in the hyperscaling relation.

\subsection{Analysis in the conformal phase}

In this subsection, we study SU(3) gauge theory with $N_{f}=12$ fundamental fermions. In the context of the SD equation with the improved ladder approximation, this theory is in the conformal window. The top panel of Fig. 1 1 shows values of $m_{P} / \Lambda$ (horizontal axis) for various values of $m_{0} / \Lambda$ (vertical axis) and $L \Lambda$, which were obtained from numerical calculation of the finite-volume SD equation. Note that we can obtain data only for $m_{P} / \Lambda>O(0.1)$ because the infrared cutoff coming from the size of space-time exists. We use these data to plot $m_{P} L$ as a function of $x$ to see whether the finite-size hyperscaling relation is satisfied, and if it does, what is the value of $\gamma$ obtained from the analysis. In the bottom of Fig. 10 finite-size hyperscaling plots for SU(3) gauge theory with 12 fundamental fermions, in which $m_{P} L$ is plotted for various values of $x$ and $L \Lambda$. The left panel, which is the case of $\gamma=0.5$, shows good alignment of data, meanwhile, the right panel, which is the case of $\gamma=0.8$, shows poor alignment compared to the case of $\gamma=0.5$. We should note that, in the framework of the ladder SD equation analysis, the value of the mass anomalous dimension at the IRFP for $N_{f}=12 \mathrm{SU}(3)$ gauge theory is $\gamma_{m}^{*}=1-\sqrt{1-\alpha_{*} / \alpha_{\mathrm{cr}}} \simeq 0.8$. Here, $\alpha_{\mathrm{cr}}=\frac{\pi}{3 C_{2}}$. Therefore, naive expectation is that the plot should show good scaling behavior for $\gamma=0.8$, rather than for $\gamma=0.5$. This shift of $\gamma$ actually comes from the correction to the hyperscaling relation due to the violation of the infrared conformality caused by the introduction of non-zero fermion 

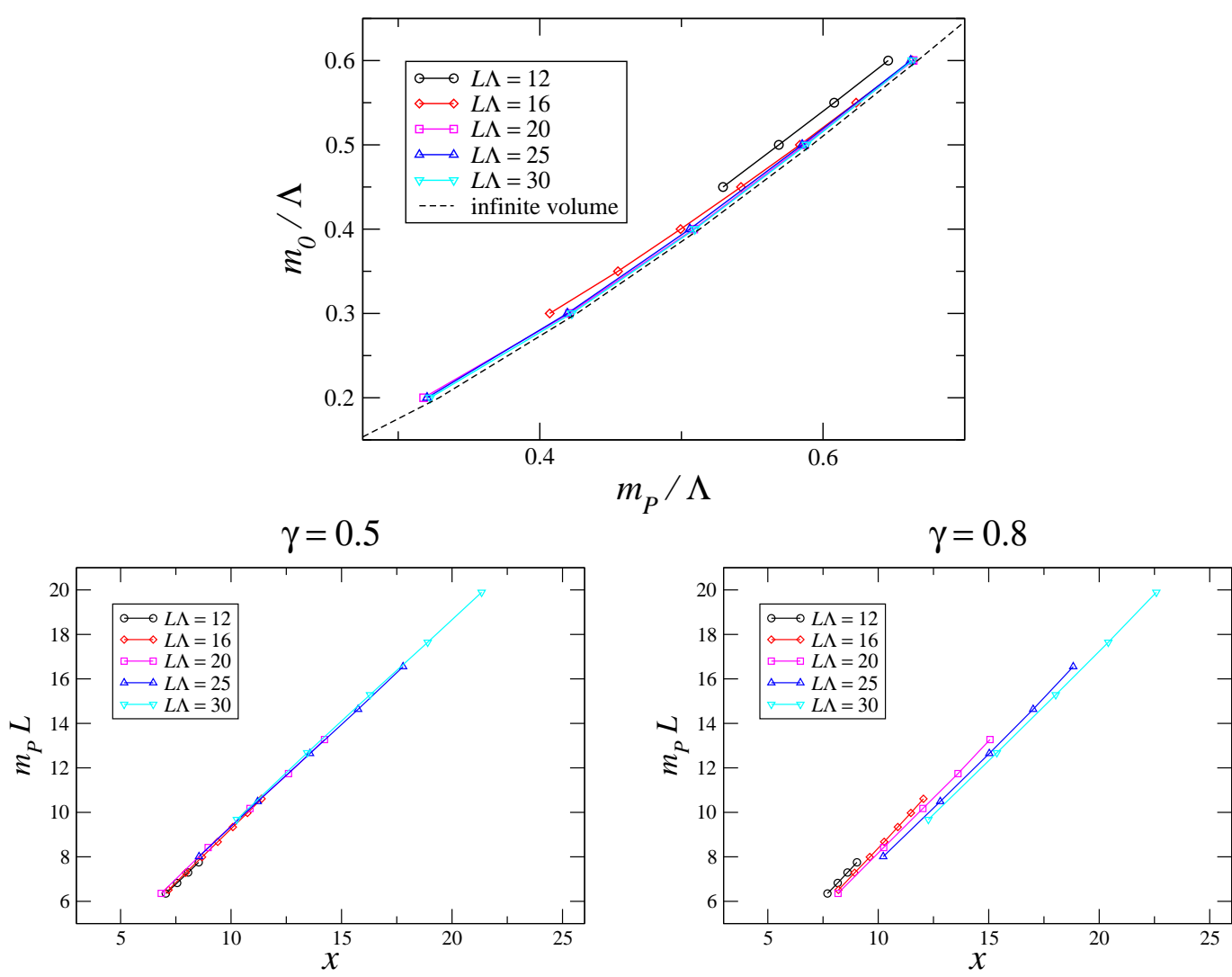

Figure 1: Top: Values of $m_{P} / \Lambda$ (horizontal axis) for various values of $m_{0} / \Lambda$ (vertical axis) and $L \Lambda$ (indicated by different symbols) for SU(3) gauge theory with 12 fundamental fermions. Dashed curve is $m_{P}$ as a function of $m_{0}$ which is obtained from the numerical solution of the SD equation in the infinite space-time. Bottom: Finite-size hyperscaling plots for SU(3) gauge theory with 12 fundamental fermions for the case of $\gamma=0.5$ (left panel) and 0.8 (right panel), respectively

mass [7]. This can be understood from the relation between $m_{0}$ and $m_{P}$ which is obtained from the analytic solution of the SD equation:

$$
\frac{m_{0}}{\Lambda}=\xi\left[\frac{\Gamma\left(1-\gamma_{m}^{*}\right)}{\Gamma\left(\frac{2-\gamma_{*}}{2}\right)^{2}}\left(\frac{m_{P}}{\Lambda}\right)^{1+\gamma_{m}^{*}}+\frac{\Gamma\left(-1+\gamma_{m}^{*}\right)}{\Gamma\left(\frac{\gamma_{*}}{2}\right)^{2}}\left(\frac{m_{P}}{\Lambda}\right)^{3-\gamma_{m}^{*}}\right] .
$$

It is obvious that if we drop the second term in the RHS of this equation, it reduces to the hyperscaling relation in the infinite volume:

$$
M \sim m_{0}^{1 /\left(1+\gamma_{m}^{*}\right)} .
$$

Therefore, the second term should be identified as the leading correction to the hyperscaling relation. The correction is large for a large value of $m_{P} / \Lambda$. In Fig. 2 we plot the effective mass anomalous dimension, $\gamma_{m}^{\text {eff }}$, which includes effect of this mass correction. (See Ref. [7] for more detailed explanation.) Note again that since the significance of the correction term is different for different values of $m_{P}$, the effective mass anomalous dimension becomes a function of $m_{P}$. In Fig. 2. together with $N_{f}=12$ case, $\gamma_{m}^{\text {eff }}$ for $N_{f}=13,14,15$ and 16 are also plotted. From this figure, 


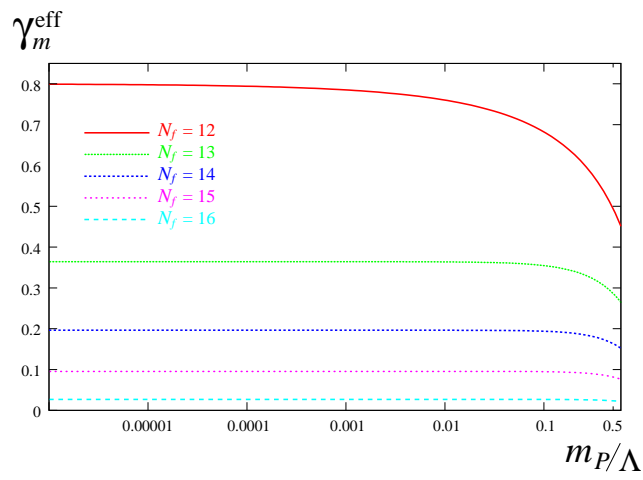

Figure 2: Effective mass anomalous dimension as a function of $m_{P} / \Lambda$ for $\mathrm{SU}(3)$ gauge theories with 12, 13, 14,15 and 16 fundamental fermions.

we can understand the fact that the hyperscaling plot in Fig. 11 shows good alignment for $\gamma_{m}=0.5$. It is because the data used for the analysis is those with rather heavy input masses. (Remember that $m_{P} / \Lambda>O(0.1)$.)

\subsection{Analysis in the broken phase}

Here, by the same procedure used in the previous subsection, we study the finite-size hyperscaling relation in theories with spontaneous chiral symmetry breaking. In the case of theories with spontaneous chiral symmetry breaking, mass gap exists even in the chiral limit, and therefore an IRFP is only approximate. Here, we show two examples: one is SU(3) gauge theory with $N_{f}=9$, and the other is that with $N_{f}=11$. The former is an example of a theory which is far away from the conformal window, in which the infrared conformality is expected to be largely violated. The latter is an example of a theory which resides close to the conformal window, and the breaking of the infrared conformality due to the spontaneous chiral symmetry breaking is expected to be small.

In Fig. 3, we show the hyperscaling plots for SU(3) gauge theory with 9 fundamental fermions. As we expected, since the infrared conformality is largely broken due to the spontaneous chiral symmetry breaking, large violation of hyperscaling relation is observed. Note that the dynamically generated mass for $N_{f}=9$ is $m_{D} / \Lambda \simeq 0.58$, where $m_{D}$ is the value of $m_{P}$ obtained by the spontaneously broken solution of the ladder SD equation in the chiral limit $m_{0} \equiv 0$. This is compared with the typical values in Fig. 3. $m_{P} / \Lambda=0.58-0.77$ for $L \Lambda=30$.

A similar plot for SU(3) gauge theory with 11 fundamental fermions is given Fig. 4. We show the result for $\gamma=1.0$, with which we found data are best aligned each other. Again, as we expected, since the theory is close to the chiral restoration point, and the effect of the spontaneous chiral symmetry breaking is small, the violation of hyperscaling relation is small. Note that $m_{D} / \Lambda \simeq 0.05$ for $N_{f}=11$, while typical values of $m_{P}$ in Fig. 4 are $m_{P} / \Lambda=0.28-0.69\left(\gg m_{D} / \Lambda\right)$ for $L \Lambda=30$. Of course, one can see that there is a small amount of misalignment. However, let us imagine those were data obtained from lattice simulations, and each data point has, say, a few percent error bar, in which case, the data might look consistent with conformal hyperscaling. Therefore, when one obtained data which look consistent with conformal hyperscaling with a large mass anomalous dimension, there is a possibility that the theory is exactly the one the technicolor model 

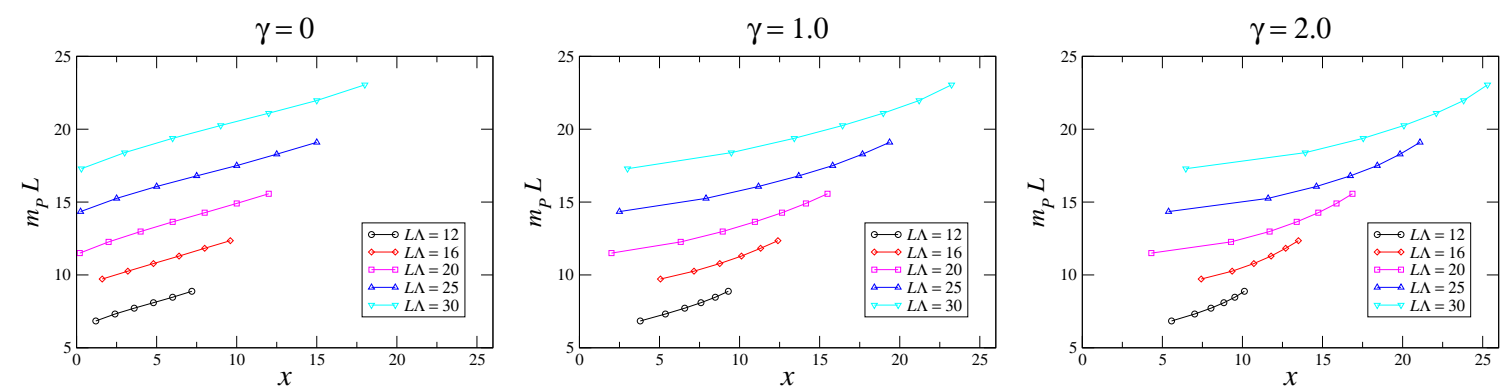

Figure 3: Values of $m_{P} L$ obtained from the finite-volume SD equation as a function of $x \equiv L \Lambda\left(m_{0} / \Lambda\right)^{1 /(1+\gamma)}$ for $\gamma=0,0.5,1.0,1.5$ and 2.0 in $\mathrm{SU}(3)$ gauge theory with 9 fundamental fermions. Data for $L \Lambda=$ $12,16,20,25$ and 30 are plotted as different symbols.

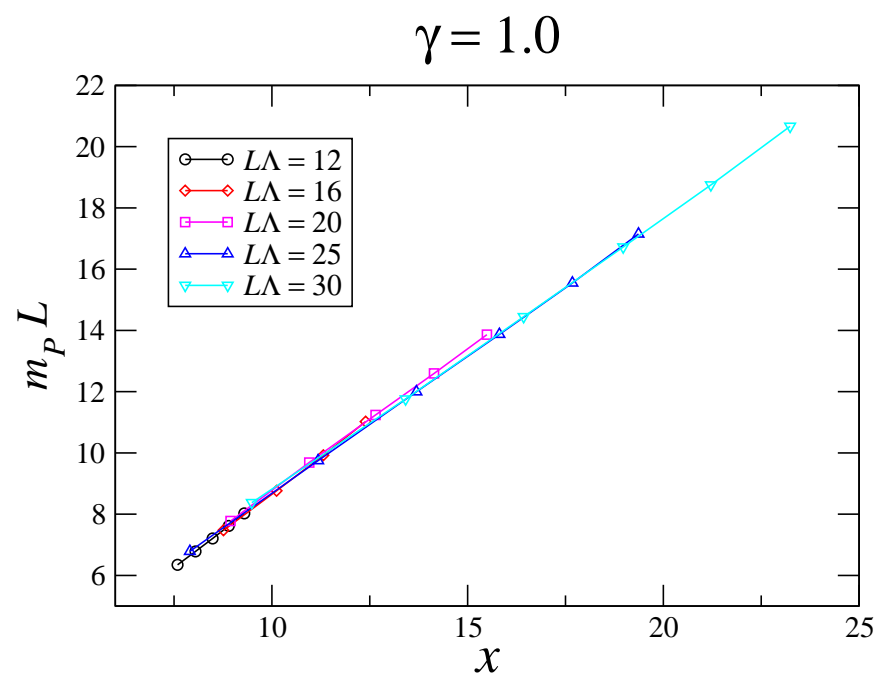

Figure 4: Values of $m_{P} L$ obtained from the finite-volume SD equation as a function of $x \equiv L \Lambda\left(m_{0} / \Lambda\right)^{1 /(1+\gamma)}$ for $\gamma=1.0$ in $\mathrm{SU}(3)$ gauge theory with 11 fundamental fermions. Data for $L \Lambda=12,16,20,25$ and 30 are plotted as different symbols.

favors, namely the dynamics with spontaneous chiral symmetry breaking at hierarchically small scale compared to $\Lambda$ with large anomalous dimension.

\section{Summary and Discussion}

We formulated the SD equation in a finite-size space-time for the study of the correction to the finite-size hyperscaling relation. By using data obtained from the finite-volume SD equation, we showed that the anomalous dimension, when identified through the finite-size hyperscaling relation neglecting the mass corrections as is often done in the lattice analyses, yields a substantially lower value of the mass anomalous dimension than its value at the IRFP. From the analytical expression of the solution of the ladder SD equation, we identified the form of the leading correction to the hyperscaling relation. We also applied the finite-volume SD equation to the chiral-symmetrybreaking phase and found that when the theory is close to the critical point such that the dynamically 
generated mass is much smaller than the explicit breaking mass, the finite-size hyperscaling relation is still operative.

Here, we summarize what we learned from the present study for different cases.

Case \#1: When the theory is deep in the hadronic phase, hyperscaling relation is totally violated, therefore, there is no confusion.

Case \#2: When the theory is in the hadronic phase, but close to the edge of the conformal window, one might observe approximate scaling behavior, and conclude that the theory is IR conformal.

Case \#3: When the theory is in the conformal window, and data are taken in a wide range of input bare mass, one might observe misalignment in the hyperscaling plot because effective mass anomalous dimension is different for different mass regions.

Case \#4: When the theory is in the conformal window, and data are taken in a rather small range of large bare mass region, one might observe good alignment in the hyperscaling plot, and obtain an effective value of $\gamma_{m}$. However, it is very possible that mass corrections to the hyperscaling relations for different physical quantities are different, so one might obtain non-universal values of $\gamma_{m}$ for hyperscaling plots with different physical quantities. Therefore, when the lattice data show a good scaling for each physical quantity, but inconsistent values of $\gamma$ (which means global fit with universal value of $\gamma_{m}$ gives bad $\chi^{2} /$ dof), it is worth doing fitting with a universal $\gamma_{m}+$ SD-inspired mass correction term for each physical quantity. It could significantly reduce $\chi^{2} /$ dof, which actually happened when we analyzed our lattice data for $N_{f}=12 \mathrm{SU}(3)$ gauge theory [9].

\section{References}

[1] S. Weinberg, Phys. Rev. D 19, 1277 (1979); L. Susskind, ibid. D 20, 2619 (1979); see also S. Weinberg, Phys. Rev. D 13, 974 (1976).

[2] See for reviews, e.g., E. Farhi and L. Susskind, Phys. Rept. 74, 277 (1981); C. T. Hill and E. H. Simmons, Phys. Rept. 381, 235 (2003) [Erratum-ibid. 390, 553 (2004)] [arXiv:hep-ph/0203079], and references therein.

[3] K. Yamawaki, M. Bando, and K. Matumoto, Phys. Rev. Lett. 56, 1335 (1986); M. Bando, K. -i. Matumoto, and K. Yamawaki, Phys. Lett. B178 (1986) 308; M. Bando, T. Morozumi, H. So, and K. Yamawaki, Phys. Rev. Lett. 59, 389 (1987).

[4] See also B. Holdom, Phys. Lett. B 150, 301 (1985); T. Akiba and T. Yanagida, Phys. Lett. B 169, 432 (1986); T.W. Appelquist, D. Karabali, and L.C.R. Wijewardhana, Phys. Rev. Lett. 57, 957 (1986).

[5] L. Del Debbio and R. Zwicky, Phys. Rev. D82, 014502 (2010). [arXiv:1005.2371 [hep-ph]].

[6] T. Appelquist, J. Terning, and L. C. R. Wijewardhana, Phys. Rev. Lett. 77, 1214 (1996); T. Appelquist, A. Ratnaweera, J. Terning, and L. C. R. Wijewardhana, Phys. Rev. D 58, 105017 (1998).

[7] Y. Aoki, T. Aoyama, M. Kurachi, T. Maskawa, K. -i. Nagai, H. Ohki, A. Shibata, K. Yamawaki and T. Yamazaki, Phys. Rev. D 85, 074502 (2012) [arXiv:1201.4157 [hep-lat]].

[8] M. Harada, M. Kurachi, and K. Yamawaki, Phys. Rev. D 68, 076001 (2003) [hep-ph/0305018]; M. Kurachi and R. Shrock, JHEP 0612, 034 (2006) [hep-ph/0605290].

[9] Y. Aoki, T. Aoyama, M. Kurachi, T. Maskawa, K. -i. Nagai, H. Ohki, A. Shibata, K. Yamawaki and T. Yamazaki, Phys. Rev. D 86, 054506 (2012) [arXiv:1207.3060 [hep-lat]]. 